\title{
Die Ranunculaceae der Flora von Zentraleuropa - Einführung: Die Geschichte des Hegi, Illustrierte Flora von Mitteleuropa ${ }^{1}$
}

\author{
Eckehart J. Jäger, Martin-Luther-Universität 06108 Halle, Neuwerk 21 \\ e-mail: jaegerbot@gmail.com
}

Der „Hegi“, die größte Flora des deutschsprachigen Raumes in Mitteleuropa, war das Werk des Pfarrer-Sohnes Gustav Hegi, geboren am 13.11.1876 in Rickenbach bei Winterthur im Kanton Zürich, gestorben am 23.4.1932 in der Gemeinde Küsnacht im selben Kanton. Am Gymnasium in Winterthur wurde Gustav von Robert Keller für die Botanik gewonnen, an der Universität Zürich wurde dieses Interesse durch Hans Schinz vertieft. Schinz und Keller verfassten zusammen die im Verlag A. Raustein (Zürich) erschienene „Flora der Schweiz zum Gebrauche auf Exkursionen, in Schulen und beim Selbstunterricht“, die 1909 erschien, 1914 um einen kritischen Band erweitert wurde und bis 1923 vier Auflagen erlebte. Zu dieser Flora leistete auch Gustav Hegi ständig Beiträge.

Nach der Promotion an der Universität Zürich im Jahr 1900 ging er nach München, war dort von 1902-1908 unter dem Morphologen Karl Goebel Kustos des Botanischen Gartens, habilitierte sich 1905 und wurde 1910 außerordentlicher Professor an der Universität München. Schon 1905 publizierte er seine farbig illustrierte „Alpenflora“, die einen sehr großen Erfolg hatte: 25 Auflagen erschienen bis 1977, zuletzt im Verlag Paul Parey zusammen mit H. Merxmüller und H. Reisigl. Noch jetzt wird sie im Buchhandel angeboten.

Sein Münchener Verleger Julius Friedrich Lehmann regte Gustav Hegi an, eine ähnliche Flora für ganz Mitteleuropa zu verfassen. Die erste Lieferung dieses großen Werkes (im vollen Titel „Illustrierte Flora von Mittel-Europa, mit besonderer Berücksichtigung von Deutschland, Oesterreich und der Schweiz zum Gebrauche in den Schulen und zum Selbstunterricht") erschien schon 1906, und bis 1931 publizierte Hegi die gesamte erste Auflage. Geplant waren zunächst sieben Bände (inkl. Registerband), durch Unterteilung wurden daraus schon in der ersten Auflage 13 Bände mit

\footnotetext{
${ }^{1}$ Zitierempfehlung: Jäger, E. J. 2020: Die Ranunculaceae der Flora von Zentraleuropa - Einführung: Die Geschichte des Hegi, Illustrierte Flora von Mitteleuropa. - Publiziert unter http://www.flora-deutschlands.de/ranunculaceae.html
} 
zusammen 7800 Seiten. Etwa zwei Drittel davon bearbeitete Hegi allein, einige Bände übernahmen Albert Thellung (Zürich, 1881-1928, Band IV/1: Berberidaceae, Lauraceae, Papaveraceae, Brassicaceae, Resedaceae) und Helmut Gams (Innsbruck, 1893-1976, Band IV/3: Fabaceae, Oxalidaceae, Geraniaceae, Tropaeolaceae und Band V/1: 25 Familien, Linaceae bis Violaceae). Den Band V/4 (Lamiaceae und Solanaceae) verfassten Hegi und Gams gemeinsam. Am Band VI/1 wirkten Albert Thellung und Emil Schmid (Zürich, 1891-1982) mit. Heinrich Marzell (Gunzenhausen, 1885-1970) lieferte für das ganze Werk die vielen volkstümlichen Namen aus den Ländern des Hegi-Gebietes. An Band V/3 war Herbert Beger (Dresden, 1889-1955, nicht Berger!) beteiligt. Die vollständige 1. Auflage ist im Internet frei zugänglich.

Hegi starb kurz nach der Vollendung der ersten Auflage der Flora im April 1932. Schon 1926 hatte er alle Ämter aus gesundheitlichen Gründen niedergelegt. (Er war 1910 Schweizer Konsul und von 1910-1920 auch Generalkonsul der Schweiz in München.)

Schon bald wurde eine Neubearbeitung nötig. Von der zweiten Auflage erschienen die Bände 1 und 21936 und 1939 noch in J. F. Lehmanns Verlag. Ganz fertig wurde die Neubearbeitung bis heute nicht. Die Bände IV/3 und V/1-4 wurden nur nachgedruckt und mit Nachtrag versehen, von den fünf Teilbänden von Band VI fehlt noch Band VI/2C (Dipsacaceae). Band VI/4 wurde nur überarbeitet und erweitert. Fünf von schließlich geplanten 25 Teilbänden wurden von 1966 bis 1998 schon in völlig neu bearbeiteter dritter Auflage publiziert: Band I/1-3, Band II/1 (von 4 Teilbänden) und Band IV/2A (von 3 Teilbänden), zwei Bände in überarbeiteter und erweiterter Auflage: Band III/1 (von 3 Teilbänden) und Band IV/1 (von 3 Teilbänden). Eine völlige Neubearbeitung der vier Teilbände von Band $V$ war zunächst nicht vorgesehen. Von den fünf Teilbänden von Band VI erschien in neu bearbeiteter 3. Auflage nur eine Lieferung von Band VI/1A (Orobanchaceae, 2009). Die zweite und dritte Auflage überschnitten sich also zeitlich, für einen Bibliothekar eine komplizierte Aufgabe.

Das Schicksal des Bandes III/3, das zu der vorliegenden Online-Publikation geführt hat, wird am Ende dieses Aufsatzes geschildert.

Gerhard Wagenitz schrieb schon 2007 (Vorwort zu Band VI/2A) von einer "leidvollen Geschichte“ des Hegi. Dafür sind mehrere Ursachen verantwortlich:

Ein Alleinstellungsmerkmal des Hegi unter den großen Floren war seine sehr breite, enzyklopädische Konzeption, die über die anderer Länderfloren weit hinausgeht. Eine Länderflora enthält gewöhnlich die Bestimmungsschlüssel für die Familien, Gattungen und Arten der in einem 
Land vorkommenden Pflanzen, ihre wissenschaftlichen Namen, Synonyme und Volksnamen, die morphologische Beschreibung sowie die Verbreitungs- und Standortsangaben. Der Hegi hatte nicht nur die Aufgabe, die unvollständig gebliebene und größtenteils überholte „Synopsis der mitteleuropäischen Flora“ von Ascherson und Graebner (1896-1939, 16 Bände) zu ersetzen und die Variabilität der Arten taxonomisch besser zu bewerten. Nein, im Hegi sollte das gesamte Wissen über die Pflanzen zusammengetragen werden: alle bekannten Tatsachen über die Verwandtschaft, Variabilität, Untergliederung und Bastardierung der Pflanzensippen, die Etymologie, Aussprache und Betonung der wissenschaftlichen Namen, die volkstümlichen Namen in den Ländern des Gebietes, die Erwähnung in historischen Schriften wie Theophrast, Hippokrates oder Plinius, die Entwicklungsgeschichte und die Biologie der Pflanzen, alle Besonderheiten der Fortpflanzung und Ausbreitung, das weltweite Areal und die horizontale und vertikale Verbreitung im Gebiet, die Geschichte der Einführung von Neophyten, die makrofossil oder palynologisch belegte Arealgeschichte heimischer Gehölze, die Verwendung und Kultur von Nutz- und Zierpflanzen, die Inhaltsstoffe der Heil- und Giftpflanzen, die anatomische Struktur, die Krankheiten und Schädlinge und immer neue Spezialgebiete wie die Pflanzensoziologie, Zytogenetik, Palynologie, die Ergebnisse der DNA-basierten Verwandtschaftsforschung und die Phylo- und Biogeographie. Anders als übliche Länderfloren enthält der Hegi auch im Laufe der Auflagen immer umfangreicher werdende Darstellungen der Pflanzenfamilien. Gerade der Berücksichtigung aller dieser Daten „hat ja u.a. das Werk seine große Beliebtheit und Verbreitung weit über die Grenzen Mitteleuropas hinaus zu verdanken." (Rechinger im Vorwort zu Band III/1 1958). Der erste Band wurde auch ins Japanische übersetzt.

Dieser breiten Konzeption steht aber die zunehmende Spezialisierung der Pflanzenforscher und ihre Konzentration auf moderne Methoden und Modell-Pflanzen gegenüber, die bevorzugte Förderung moderner Richtungen gegenüber der „klassischen“ Botanik und die abnehmende Kenntnis der Pflanzen im Freiland. Dadurch wurde die Suche nach kompetenten Autoren, die ein gut illustriertes Manuskript termingerecht liefern, immer schwieriger.

Die Verwirklichung des breiten Konzeptes war nur durch die Zusammenarbeit mit zahlreichen Kollegen möglich. Im Band VI/3 S.VII werden 21 Spezialisten für einzelne Gebiete genannt. Zunächst sahen es prominente Vertreter der Botanik noch als Ehre an, an dem renommierten Werk mitzuarbeiten: K.-H. Rechinger (Wien, 1906-1998, „Wirklicher geheimer Rat und erster Direktor des Naturhistorischen Museums"; Herausgeber von Band III/1 1958 und Band III/2 1979), Hermann Meusel (Nationalpreisträger, Halle 1909-1997; mit H. Mühlberg Bearbeiter der Nymphaeaceae in Band III/3 2. Auflage 1974 und mit K. Werner der Silenoideae in Band III/2 
2. Auflage 1978), auch noch Friedrich Ehrendorfer (Wien, * 1927; Band VI/2B 2. Auflage Rubiaceae 2016, mit A. Kästner). An der 2. Auflage von Band 1 (Farnpflanzen, 1984) war sogar ein Nobelpreisträger beteiligt: Tadeus Reichstein (1897-1996, Zürich, Basel), der sich im hohen Alter noch in über 100 Publikationen mit der Systematik der Farne beschäftigte, nachdem er vorher die Nebennierenrindenhormone erforscht und die Vitamin-C-Herstellung entwickelt hatte (Nobelpreis 1950). Aber in den 70er Jahren fand der Carl Hanser Verlag, in dem der Hegi nach langer Pause in den Kriegs- und Nachkriegsjahren seit 1957 erschien und dessen Vertreter uns in Halle besuchte, kaum noch Autoren für die Fortführung der 2. und 3. Auflage. Mehrere Manuskripte blieben unvollständig.

Ein idealer Autor einer Hegi-Bearbeitung war schwer zu finden, denn er sollte, wie Gerhard Wagenitz ironisch schildert, über einen leichten Zugang zur gesamten einschlägigen Literatur und zu allen wichtigen Herbarien verfügen, also unbegrenzte Zeit und Reisemittel zur Verfügung haben, die Pflanzen im (botanischen) Garten kultivieren und beobachten können, über ein Labor für anatomische, zytogenetische, biochemische Untersuchungen und DNA-Analysen verfügen und einen guten Zeichner beschäftigen können.

Aus dem enzyklopädischen Charakter des Hegi ergab sich aber auch ein ständig wachsender Umfang. Nicht nur die Kenntnis der Sippen nahm schnell zu. Beispielsweise wurden im Band II in der ersten Auflage (1909) für Galanthus nur vier Arten anerkannt, heute sind es 21, für Gagea wurden immerhin schon mehr als 50 Arten genannt, heute sind nach "Plants of the World" 211 Arten akzeptiert. Bei Alchemilla wuchs die Artenzahl im Hegi von sieben in der 1. Auflage auf 137 in der 2. Auflage an. Vor allem waren aber immer neue Forschungsergebnisse aufzunehmen, z.B. immer genauere Verbreitungsangaben, und im Ergebnis der Verwandtschaftsforschung und der Auswertung alter Literatur waren immer wieder geänderte wissenschaftliche Namen zu berücksichtigen.

Die Bände mussten immer stärker unterteilt werden. Schon in der 1. Auflage waren die Bände IV, V und VI in drei, vier und zwei Teilbände aufgeteilt worden, so dass aus sechs geplanten Bänden 12 (Teil-)Bände geworden waren (außer dem Registerband, der wegen der ständig geänderten Bandaufteilung nicht neu aufgelegt, sondern durch ein alphabetisches Verzeichnis der Gattungen und Familien einschließlich der Synonyme ersetzt wurde, z.B. im Anhang des Bandes VI/2B 2016). In der 2. Auflage umfasste das Werk 19 (Teil-)Bände, in der dritten waren mindestens 25 vorgesehen.

Der Hegi wurde also immer umfangreicher und teurer. Umfasste die 1. Auflage 7.800 Seiten, so war der Umfang für die 18 verfügbaren Bände (nach einer Übersicht auf dem Rücktitel von Band 
VI/2B Lfg. 3 2007) auf 10.076 Seiten angestiegen, hochgerechnet auf das ganze Werk hätte er sich mit etwa 15.000 Seiten gegenüber der ersten Auflage verdoppelt.

Der Preis lag zuletzt für die 18 verfügbaren Bände bei 2359,55 €, hochgerechnet für das ganze Werk bei über $3000 €$. Anfangs durfte der Hegi in keiner Schulbibliothek fehlen, ja jeder BiologieLehrer sollte ihn besitzen. Im Verlag Paul Parey dachte man später an eine billige Ausgabe in hoher Stückzahl, einen „Volks-Hegi“. Das wurde nicht realisiert, die Insolvenz des Verlages hat damit nichts zu tun. Von der im Weißdorn-Verlag erschienenen 2. Auflage des Bandes VI/2B (Rubiaceae, 2016, 500 Exemplare) setzte der Verleger in den ersten beiden Jahren nur die Hälfte ab.

Ein anderes Problem entstand für die Abgrenzung des Gebietes der Flora durch die Folgen der beiden Weltkriege. Das Gebiet der Flora entsprach vor dem ersten Weltkrieg dem deutschen Kaiserreich von 1871, der Schweiz, Österreich (ohne Ungarn und Bosnien-Herzegowina, aber einschließlich Süd-Tirol, Böhmen und Mähren, sowie Krain und Küstenland, die jetzt zu Slowenien und Kroatien gehören). Es umfasste also auch Elsass-Lothringen, Ober- und Nieder-Schlesien, Pommern, Ost- und Westpreußen. Ähnlich ist es noch auf der Karte des Gebietes im Band VI/2A (2008, Campanulaceae u.a.) und im Band VI/2B (2016, Rubiaceae) umgrenzt, aber nun einschließlich der gesamten Ost- und Mittel-Alpen bis zum Genfer See. Wenn auch die alten Grenzen weitgehend der pflanzengeographischen Umgrenzung Zentraleuropas entsprechen, so ist diese Umgrenzung aus politischen Gründen doch jetzt nicht mehr vertretbar. Eine finanzielle Förderung im Rahmen eines Forschungsauftrages war für den Hegi deshalb auch kaum zu beantragen. Erst im Rubiaceenband wurde ganz Polen berücksichtigt.

Schließlich machte auch der mehrfache Wechsel des Verlages die Produktion des Werkes nicht leichter. Mit der Wahl seines Verlegers Julius Friedrich Lehmann (1864-1935) hatte Gustav Hegi keine glückliche Hand. Nach anfänglicher erfolgreicher Herausgabe medizinischer Literatur wandte sich Lehmann schon 1905 der Rassenlehre zu. Er gehörte seit 1910 der deutschen Gesellschaft für Rassenhygiene an und brachte nun Bücher und Zeitschriften auf den Markt, die zur Herausbildung der nationalsozialistischen Ideologie beitrugen. In Band V des Hegi (1. Auflage 1928) sind Anzeigen aus dem Verlag eingefügt: „Rasse und Seele“, „Deutsche Rassenbilder", „Rassenkunde Europas“, „Ist Rasse Schicksal?“, „Volk und Rasse“, „Der Kulturumsturz - die Drohung des Untermenschen“. Im letzteren Titel schildert L. Stoddard die „Überschwemmung seiner amerikanischen Heimat durch einwandernde Scharen minderrassischer Fremder" und die "bolschewistische Auflehnung der primitiven Menschen gegen die Kultur", und Lehmann hofft, "dass das deutsche Volk dem Fremden vielleicht leichter glauben wird als den eigenen Volksgenossen“. Lehmann publizierte die Monatsschrift "Volk und Rasse“, deren Heraus- 
geber Heinrich Himmler war. Viele der in Lehmanns Verlag erschienenen Schriften wurden durch die Münchener Reichswehr-Führung für den „Truppenaufklärungsdienst" gekauft (Wikipedia 2020). 1920 trat Lehmann der NSDAP bei und unterstützte sie regelmäßig durch hohe finanzielle Überweisungen (auch an Adolf Hitler). Die von ihm erworbene Burg Hoheneck bei Ipsheim stellte er für "nationale Schulungswochen“ und als Stützpunkt der SA zur Verfügung. Zu seinem 70. Geburtstag erhielt er das goldene Partei-Abzeichen und wurde mit dem „Adlerschild des deutschen Reiches", dem höchsten Wissenschafts-Preis, ausgezeichnet. Nach dem Ende des Krieges war auch der Verlag am Ende, er kam unter Treuhand-Verwaltung.

Der Hegi wurde nach 18 Jahren Pause in der Kriegs- und Nachkriegszeit von 1957 bis 1974 im ebenfalls in München ansässigen Carl Hanser Verlag weitergeführt. Dort war das Werk ein Fremdkörper, denn trotz eines weiten Publikations-Spektrums (Wirtschaft, Technik, Belletristik, Kinderbücher) produzierte Hanser sonst keine naturwissensschaftliche Literatur.

Die zweite Auflage des Hegi sollte mit der vollständigen Neubearbeitung der Bände II-IV und VI fortgesetzt werden. Band V mit vier Teilbänden war nicht zur Neubearbeitung vorgesehen. Die vier Teilbände erschienen 1964 (Teil 4: Lamiaceae, Solanaceae) und 1966 (Teil 1: Linaceae bis Violaceae, Teil 2: Cactaceae bis Apiaceae, Teil 3: Ericaceae bis Boraginaceae) als Nachdrucke mit Nachtrag, ebenso Band IV/3 (Leguminosen, Oxalidaceae, Geraniaceae, Tropaeolaceae 1964). Von der zweiten Auflage erschienen im Carl Hanser Verlag sechs völlig neu bearbeitete Bände:

- Band III/1 1957-1958, Juglandaceae, Salicaceae, Betulaceae, Fagaceae bis Polygonaceae; Herausgeber und Bearbeiter K.-H. Rechinger (Wien, 1906-1998), Mitarbeiter Annelis Schreiber (München), L. Hoerhammer (München, Inhaltsstoffe), H. Marzell (volkstümliche Namen), H. Meusel (Halle, Verbreitung), E. Oberdorfer (Standortsökologie, Pflanzensoziologie), A. Patzak, F. Petrak, M. v. Rochow,

- Band III/2 1959-1971, Phytolaccaceae, Amaranthaceae, Chenopodiaceae, Illecebraceae, Caryophyllaceae, Portulacaceae; Herausgeber K.-H. Rechinger, Bearbeiter P. Aellen (Schaffhausen, Basel, 1896-1973), H.-C. Friedrich (München), H. Meusel (Halle, 1909-1997), H. Mühlberg (Halle, *1932) und K. Werner (Halle, 1928-2003); von 1959-1971 sieben Lieferungen, dieser Band wurde mit den Lieferungen 8-10 erst 1979 im Verlag Paul Parey abgeschlossen,

- Band III/ 3 Nymphaeaceae, Ceratophyllaceae, Paeoniaceae, Ranunculaceae; s. unten.

- Band IV/1 1963, Berberidaceae, Lauraceae, Papaveraceae, Brassicaceae, Resedaceae; Herausgeber und Bearbeiter F. Markgraf (Zürich, 1897-1987), Mitarbeiter L. Hörhammer 
(München; Phytochemie), H. Marzell (Gunzenhausen, 1895-1970; volkstümliche Pflanzennamen), E. Oberdorfer (Freiburg, 1905-2002; Standort, Pflanzensoziologie), H. Straka (Kiel, 1920-2009 Palynologie, Paläobotanik) und R. Wannenmacher (Wien, Inhaltsstoffe),

- Band IVI2A 1961-1966, Droseraceae, Philadelphaceae, Grossulariaceae, Crassulaceae, Saxifragaceae, Parnassiaceae, Rosaceae 1. Teil, Herausgeber und Bearbeiter Herbert Huber (Würzburg, Kaiserslautern, 1931-2005), Mitarbeiter H. Marzell (volkstümliche Pflanzennamen), E. Oberdorfer (Standort, Pflanzensoziologie), H. Straka (Kiel 1920-2009; Palynologie, Paläobotanik) und R. Wannenmacher (Wien; Inhaltsstoffe),

- Band VI/1 1965-1974, Scrophulariaceae, Orobanchaceae, Lentibulariaceae, Plantaginaceae erschien in der völlig neu bearbeiteten 2. Auflage in acht Lieferungen, Herausgeber D. Hartl (Mainz, 1927-2015) und G. Wagenitz (Berlin, Göttingen 1927-2017), außerdem die ersten Lieferungen von Band VI/2A (Lfg. 1 und 2 von 4, Rest im Weißdorn-Verlag 2008) und von Band VI/3 (Lfg. 1-4, 5. Lfg. im Verlag Paul Parey).

Die dritte Auflage wurde im Carl Hanser Verlag fortgesetzt mit Band II/1, Cyperaceae bis Juncaceae, Lfg. 1-3 1966-1969 (Lfg. 4-6 1977-1980 im Verlag Paul Parey), Herausgeber W. Schultze-Motel (Berlin, 1934-2011), Bearbeiter R. Kandeler (Wien, 1927-2015), U. und D. Müller-Doblies (Berlin, beide *1938), D. Podlech (München, *1937), H. Riedl (Wien, *1936) und W. Schultze-Motel.

Am 1. Juli 1975 erfolgte die Übernahme des Werkes durch den Verlag Paul Parey Berlin-Hamburg, der auf biologische und land- und forstwissenschaftliche Literatur spezialisiert war. Die bisher blaugrauen Bände und Lieferungen erschienen nun in dunkelgrünem Leinen mit gelbgrünem Schutzumschlag, die Lieferungen in gelbgrünem Karton.

Gesamt-Herausgeber waren zunächst (bis 1990) Hans Joachim Conert (Frankfurt/M., *1929), Ulrich Hamann (Bochum, 1931-1990), Wolfram Schultze-Motel (Berlin, 1934-2011) und Gerhard Wagenitz (Göttingen, 1927-2017).

Ulrich Hamann sollte die Bände II/2-4 in der dritten Auflage herausgeben (zusammen mit H. Meusel); E. J. Jäger hatte ihm dafür schon Verbreitungsangaben und Karten zur Verfügung gestellt. U. Hamanns früher Tod ist der Grund dafür, dass bis heute keine Neubearbeitung vorliegt. Im Paul Parey Verlag erschienen fünf völlig neu bearbeitete Bände:

- Band I/1 1984 3. Auflage Farnpflanzen, Herausgeber K. U. Kramer (Zürich, *1928), Bearbeiter J. Dostál (Prag, 1903-1999) und T. Reichstein (Zürich, Basel; 1897-1996), Mitarbeit von K. U. Kramer und C.-R. Fraser-Jenkins (London, *1948), 
- Band I/2 1981 3. Auflage Gymnospermen, Monokotyledonen: Alismataceae bis Scheuchzeriaceae; Herausgeber F. Markgraf (Zürich, 1897-1987), Bearbeiter H. Zoller (Basel, 19232009) und F. Markgraf,

- Band II/1 1980 3. Auflage Cyperaceae, Typhaceae, Araceae, Lemnaceae, Juncaceae; Herausgeber W. Schultze-Motel, Bearbeiter: R. Kandeler (Wien, 1927-2015), Ute und D. Müller Doblies (Berlin, beide *1938), D. Podlech (München, *1937), H. Riedl (Wien, *1936),

- Band III/2 1979 2. Auflage Phytolaccaceae, Amaranthaceae, Chenopodiaceae, Illecebraceae, Caryophyllaceae, Portulacaceae; Herausgeber K.-H. Rechinger, s. oben unter Carl Hanser Verlag,

- Band VI/3 1979 2. Auflage Asteraceae allgemeiner Teil; Eupatorium bis Achillea; Herausgeber und Bearbeiter G. Wagenitz;

und drei Bände als überarbeiteter und erweiterter Nachdruck:

- Band III/1 1981 3. überarbeitete und erweiterte Auflage. Herausgeber G. Wagenitz, Bearbeiter V. Melzheimer (Berlin, Marburg, *1939) und H. Straka,

- Band IVI1 1986 3. überarbeitete und erweiterte Auflage. Herausgeber und Bearbeiter W. Schultze-Motel,

- Band VI/4 1987 2. überarbeitete und erweiterte Auflage, Asteraceae II: Matricaria bis Hieracium. Herausgeber und Bearbeiter G. Wagenitz.

Der Verlag Paul Parey wurde insolvent und musste den Hegi 1992 an den Blackwell Wissenschafts-Verlag Berlin verkaufen. Dort traf sich am 3.2.1997 am Kurfürstendamm in Berlin die Gruppe der Gesamtherausgeber, nun mit Hans J. Conert (Frankfurt), Eckehart J. Jäger (Halle/Saale), Joachim W. Kadereit (Mainz), Wolfram Schultze-Motel (Berlin), Gerhard Wagenitz (Göttingen) und Heinrich E. Weber (1932-2020, Osnabrück-Vechta).

Im Blackwell Wissenschafts-Verlag erschienen von 1994-1997 vier völlig neu bearbeitete Bände in dunkelgrünem Karton, der erste im Blackwell Wissenschafts-Verlag, die weiteren als Imprint Parey Buchverlag im Blackwell Wissenschafts-Verlag:

- Band I/3 Poaceae wurde mit den Lieferungen 7-10 in der 3. Auflage abgeschlossen Herausgeber und Bearbeiter H. J. Conert. Die Lieferungen 1-6 waren von 1979 bis 1992 im Verlag Paul Parey erschienen. Die Lieferung 7 (1994) erschien im Blackwell Wissenschafts-Verlag Berlin, Wien; die 8.-10. Lieferung 1996 und 1997 im Parey Buchverlag Berlin, einem Imprint des Blackwell-Verlages, das ganze Buch also in vier von sechs Verlagen des Hegi!

- Band IVI2A 1995 Platanaceae bis Parnassiaceae und Rosaceae 1. Teil, Herausgeber H. E. Weber (Osnabrück/Vechta, 1932-2020), Bearbeiter K. Adolphi (*1944, Köln), P. Gerstberger 
(Bayreuth, *1951), K. Kaplan (Metelen), W. Lippert (München, 1937-2018), H. Scholz (BerlinDahlem, 1928-2012), Ilse Scholz (*1928, Berlin) und H. E. Weber.

- Band IVI2B 1995 Rosaceae 2. Teil: Sanguisorba, Alchemilla, Cydonia, Pyrus, Malus, Sorbus, Amelanchier, Cotoneaster, Crataegus und Prunoideae; Herausgeber H. Scholz, Bearbeiter G. Dahlgren (1931-2009, Lund), S. Fröhner (Dresden, *1941), H. Kutzelnigg (*1941), W. Lippert (München, 1937-2018), F.-G. Schroeder (Göttingen, 1930-2019), R. Silbereisen (Ravenburg/ Bavendorf, 1928-2016), H. Scholz, I. Scholz (s.o.).

- Band IVI2C 2003 Rosaceae: Potentilla, Sibbaldia, Rosa; Herausgeber H. E. Weber, Bearbeiter H. Henker (Neukloster, *1930), P. Gerstberger (Bayreuth, *1951) und H. E. Weber (Osnabrück/ Vechta, 1932-2020).

2003 trennte sich Blackwell von allen biowissenschaftlichen Titeln. Der herrenlose Hegi wurde 2003 durch Vermittlung von E. J. Jäger von dem Jenenser Weißdorn-Verlag übernommen, der schon einige botanische Werke herausgebracht hatte.

Dort erschien in hellblauem Karton (die Lieferungen in hellblauer Broschur)

- Band VI/2A 2. Auflage 2008 Cucurbitaceae, Caprifoliaceae, Adoxaceae, Valerianaceae, Campanulaceae; Herausgeber G. Wagenitz, Bearbeiter H. Scholz, F. Weberling (Ulm, Erbach, 1926-2009),

- Band VI/2B 2. Auflage 2016 Rubiaceae; Herausgeber E. J. Jäger (Halle, *1934), Bearbeiter F. Ehrendorfer (Wien, *1927) und A. Kästner (Halle, *1936), und

- Band VI/1A: 3., vollständig neu bearbeitete Auflage, Lieferung 12009 Orobanchaceae; Herausgeber G. Wagenitz, Bearbeiter J. Pusch (Frankenhausen, *1962) und K.-F. Günther (Jena, *1941).

\section{Band III/3 und das Ende der Print-Ausgabe des Hegi}

Band III/3 (Paeoniaceae, Ceratophyllaceae, Nymphaeaceae, Ranunculaceae) war in der zweiten Auflage im Carl Hanser Verlag 1974 von K.-H. Rechinger (Wien, Lieferung 1) und J. Damboldt (Berlin, 1937-1978, Lfg. 2-5) herausgegeben worden. Bearbeitet wurden die Ceratophyllaceae und Nymphaeaceae von H. Meusel (Halle, 1909-1997) und H. Mühlberg (Halle, *1932), die Magnoliaceae und Paeoniaceae von W. Zimmermann (Tübingen, 1892-1980), die Ranunculaceae von W. Zimmermann (außer Ranunculus) und J. Damboldt (Berlin, 1937-1978). Die Inhalts- 
stoffe bearbeitete für Ranunculus Ch. Leuckert (Berlin, 1930-2011), die volkstümlichen Namen H. Marzell (Gunzenhausen, 1885-1970), die Pollenmorphologie und die subfossilen Pollenfunde H. Straka (Kiel, 1920-2009), die Standortsökologie und Vergesellschaftung E. Oberdorfer (Freiburg, 1905-2002).

In der 3. Auflage war für diesen Band zunächst eine überarbeitete und erweiterte Fassung der 2. Auflage vorgesehen (Nachdruck mit Ergänzungen). Von Wolfram Schultze-Motel aus der Gruppe der Gesamtherausgeber wurde 1993 die Bearbeitung Volker Melzheimer (Berlin, Marburg, *1939) angetragen, weil dieser in Berlin über die Berliner Vertreter von Ranunculus sect. Auricomus gearbeitet hatte und auch durch die Mitarbeit am Nachtrag zum Nachdruck von Band III/1 (1981) schon mit dem Hegi vertraut war. Melzheimer übernahm die Aufgabe, führte das systematische Konzept von M. Tamura (in Kubitzki et al.: The Families and Genera of Vascular Plants II, Berlin 1993) ein und überarbeitete den Text für die Einführung in die Familie und für einen großen Teil der Arten. Nachdem er sich von 1997-1999 besonders mit dem schwierigen Ranunculus auricomus-Aggregat beschäftigt hatte und dafür auf Reisen nach Polen, in die Schweiz, nach Süddeutschland, in die Südalpen und nach Österreich viele neue Erkenntnisse gewonnen hatte, konnte er im Jahr 2000 das fertige Manuskript des überarbeiteten und ergänzten Nachdrucks den Gesamtherausgebern vorlegen.

In dieser Zeit entschloss sich aber der Blackwell Wissenschafts-Verlag, den Hegi abzugeben, so dass sich die Publikation auf unbestimmte Zeit verzögerte.

Am 22.10.2004 trafen sich die Gesamtherausgeber Hans J. Conert, Eckehart J. Jäger, Joachim W. Kadereit, Gerhard Wagenitz und Heinrich E. Weber (1932-2020) beim Verleger Gerald Hirsch (Weißdorn-Verlag Jena) und besprachen das weitere Vorgehen. Das Manuskript des überarbeiteten und ergänzten Nachdrucks war jetzt in vielen Teilen nicht mehr aktuell. Daher wurde für Band III/3 nun doch eine völlige Neubearbeitung für nötig gehalten. E. J. Jäger wurde mit der Herausgabe des Bandes beauftragt. Er wandte sich an mehrere Spezialisten für verschiedene Gattungen oder Gruppen der Ranunculaceae, die den Text für ihre Gattungen übernahmen: M. Baltisberger (Zürich; Ranunculus montanus-Gruppe), W. Gutermann (Wien; Ficaria), R. Hand (Berlin; Thalictrum), E. Hörandl (Göttingen; Ranunculus außer sect. Batrachium und sect. Auricomus), H. Mühlberg (Halle; Nymphaeaceae, Ceratophyllaceae), W. K. Rottensteiner (Graz; Delphinium, Consolida, Aconitum) und G. Wiegleb (Cottbus; Ranunculus sect. Batrachium). H. Niklfeld (Wien) übernahm die Bearbeitung der wissenschaftlichen Namen, ihrer Synonyme und der Volksnamen aller Gattungen, Arten und Unterarten, trug zur Beschreibung der Verbreitung in Österreich bei und ergänzte den Abschnitt über Aquilegia. Die übrigen Gattungen bearbeitete 
E. J. Jäger. Viele waren bisher von V. Melzheimer unverändert oder wenig verändert aus der Bearbeitung von Damboldt in der 2. Auflage übernommen worden. Die Bearbeitung der allgemeinen Kapitel zu den Ranunculales und Ranunculaceae übernahm Joachim W. Kadereit (Mainz, *1956) als Spezialist für Phylogenie und Evolution. V. Melzheimer steuerte den umfangreichen Abschnitt über Ranunculus sect. Auricomus bei.

Das Erscheinen des Bandes war vom Weißdorn-Verlag schon für 2007 angekündigt worden, verzögerte sich aber, trotz intensiver Arbeit besonders in den Jahren 2008-2012. Der BandHerausgeber E. J. Jäger war auch für den Band VI/2B (Rubiaceae, erschienen 2016) verantwortlich und hatte damit bis 2015 viel Arbeit, vorher noch mit der Durchsicht und Korrektur des Bandes VI/2A (2008, vor allem Campanulaceae von D. Podlech). Als unerfüllbar erwies sich der Wunsch, einen Co-Autor für die Delphinieen zu finden. Die Bearbeitung von W. K. Rottensteiner wich vom üblichen taxonomischen Konzept stark ab und war schon von M. A. Fischer (Wien) nicht unkommentiert in seine Exkursionsflora für Österreich übernommen worden. Sie war auch nicht mit der Bearbeitung in der Flora der Schweiz kompatibel. Zuletzt hätte noch die Forderung nach der Gebiets-Erweiterung auf ganz Polen die Aufnahme weiterer Arten nötig gemacht, z.B. auch der Delphinieen aus der Tatra.

Der Verleger der letzten neu aufgelegten Hegi-Bände, Dr. Gerald Hirsch (Weißdorn-Verlag Jena), entschloss sich aber im Frühjahr 2019, u.a. wegen zunehmender Absatz-Probleme, die Arbeit an der Publikation der Hegi-Neubearbeitung ganz einzustellen. Die schon in den neunziger Jahren begonnene Arbeit an der 3. Auflage des Bandes III/3 war aber, auch dank der Hilfe der Spezialbearbeiter, so weit fortgeschritten, dass sich Joachim W. Kadereit (neben E. J. Jäger Gesamtherausgeber des Hegi) nach einem Besuch von V. Melzheimer und in Absprache mit E. J. Jäger entschloss, eine Möglichkeit der Online-Publikation für die Ranunculaceae, die größte Familie des Bandes, zu suchen. Diese Möglichkeit wurde nun bei der „Gesellschaft zur Erforschung der Flora Deutschlands“ gefunden. Dafür verdienen Ralf Hand und der übrige Vorstand der Gesellschaft großen Dank. Fast alle Autoren stimmten zu und versprachen, ein (evtl. überarbeitetes) Manuskript zu liefern. 


\section{Danksagung}

Herrn Professor Dr. Joachim W. Kadereit danke ich für die kritische Durchsicht des Manuskriptes und besonders für die Initiative zur Online-Publikation der vorliegenden Manuskripte. Frau Doris Franke, Dipl. Designerin (FH), übernahm freundlicherweise die endgültige Gestaltung und die Anfertigung des PDFs dieser Arbeit und wird dies für alle Publikationen in dieser Reihe tun. Herrn Dr. Ralf Hand danke ich herzlich für die Bemühungen um die Online-Publikation der Manuskripte auf den Webseiten der Gesellschaft zur Erforschung der Flora Deutschlands. 